\title{
Flavor symmetry breaking in mixed-action QCD
}

\author{
Oliver Bär \\ Institute of Physics \\ Humboldt University, Berlin, Germany \\ E-mail: baer@physik.hu-berlin.de

\section{Maarten Golterman*} \\ Department of Physics and Astronomy \\ San Francisco State University, San Francisco, CA 94132, USA \\ E-mail: maarten@stars.sfsu.edu

\section{Yigal Shamir} \\ Raymond and Beverly Sackler School of Physics and Astronomy \\ Tel-Aviv University, Ramat Aviv, 69978 Israel \\ E-mail: shamirepost.tau.ac.il
}

\begin{abstract}
We study the phase structure of mixed-action QCD with two Wilson sea quarks and two chiral valence quarks, starting from the chiral lagrangian. A priori, the effective theory allows for a rich phase structure, including a phase with a condensate made of sea and valence quarks. Because this would lead to mass eigenstates that are admixtures of sea and valence fields, pure-sea correlation functions would depend on valence quark masses, in contradiction with the actual setup of mixedaction simulations. Using properties of the chiral Dirac operator, we prove that such a phase does not occur, and that this leads to bounds on low-energy constants.
\end{abstract}

The XXIX International Symposium on Lattice Field Theory - Lattice 2011

July 10-16, 2011

Squaw Valley, Lake Tahoe, California

\footnotetext{
* Speaker.
} 


\section{Introduction}

In lattice QCD with a mixed action (MAQCD), the discretization of the Dirac operator in the sea sector (i.e., the operator from which the fermion determinant is constructed) is chosen to be different from the discretization of the Dirac operator in the valence sector (i.e., the operator which is inverted to obtain quark propagators attached to external lines).

The continuum limit of a mixed-action theory is a partially quenched theory [1], because even in the continuum limit the valence and sea quark masses $m_{v}$ and $m_{s}$ for each flavor do not have to be equal. This breaks unitarity already in the continuum limit, but when the lattice spacing $a \neq 0$, it is not even possible to define equality of $m_{v}$ and $m_{s}$ in a universal way [2]. This implies, of course, that unitarity is always broken at $a \neq 0$ in a mixed-action theory.

It is relatively straightforward to check the validity of the mixed-action approach in weakcoupling perturbation theory. More non-trivial, non-perturbative information about the validity is obtained by constructing the low-energy effective field theory (EFT) for MAQCD, mixed-action chiral perturbation theory (MAChPT) [月, 3, 包. Chiral perturbation theory (ChPT) gives access to the phase diagram of the lattice theory, and, as we will see, this allows for a non-trivial check of the field-theoretical definition of MAQCD and the validity of MAChPT. Here, we investigate the phase structure of MAQCD with two chiral (Ginsparg-Wilson [5]) valence quarks, and two Wilson sea quarks. For a more detailed account which includes technicalities, we refer to Ref. [6].

\section{MAChPT}

Field-theoretically, MAQCD with two (Wilson) sea and two (chiral) valence flavors is a theory with six quarks: the two sea quarks, the two valence quarks, and two more "ghost" quarks with a Dirac operator identical to that of the valence quarks, but with opposite statistics, such as to effect a cancellation between valence and ghost determinants, thus removing both valence and ghost quarks from the sea.

This implies that the non-linear field $\Sigma$ representing the pions in the EFT is a $6 \times 6$ graded matrix

$$
\Sigma=\left(\begin{array}{cc}
\exp \left(\frac{2 i}{f} \phi\right) & \bar{\omega} \\
\omega & \exp \left(\frac{2}{f} \hat{\phi}\right)
\end{array}\right),
$$

with $\phi$ and $\hat{\phi}$ hermitian matrices of size $4 \times 4$ and $2 \times 2$, respectively, and $\omega$ and $\bar{\omega}$ rectangular, Grassmann-values matrices. The matrix $\phi$ describes the pions in the sea and valence sectors, the matrix $\hat{\phi}$ those made of ghost quarks, while the Grassmann valued components describe fermionic pions made out of one ghost, and one sea or valence quark. $f$ is the pion decay constant in the chiral limit. For an overview of the precise symmetry structure of the theory, which leads to the unfamiliar way in which $\hat{\phi}$ appears in the non-linear field $\Sigma$, see for instance Ref. [7].

Since we are only interested in the phase diagram of the theory here, it suffices to consider the chiral potential, which, in simplified form, and rescaled by the combination of low-energy constants (LECs) $B_{0} f^{2} / 4$, is given by [2]

$$
V=-\operatorname{str}\left(M\left(\Sigma+\Sigma^{-1}\right)\right)-\frac{c_{1}}{4} \operatorname{str}\left(P_{s} \Sigma P_{s} \Sigma^{-1}\right)-c_{2} \operatorname{str}\left(P_{s} \Sigma^{-1} P_{s} \Sigma^{-1}+P_{s} \Sigma P_{s} \Sigma\right)
$$


in which $P_{s}=\operatorname{diag}(1,1,0,0,0,0)$ projects on the sea sector, and $M$ is the quark mass matrix

$$
M=\operatorname{diag}\left(m_{s}, m_{s}, m_{v}, m_{v}, m_{v}, m_{v}\right)
$$

where the first two $m_{v}$ entries correspond to the valence quarks, and the last two to the ghost quarks. We consider the "large cutoff effects" regime, in which the chiral power counting is such that $m_{s} \sim m_{v} \sim a^{2}$. This implies that if we consider terms in the potential to linear order in the quark masses, we should also consider all terms of order $a^{2}$, and the terms proportional to $c_{1}$ and $c_{2}$ are such terms. ${ }^{1}$ The constant $c_{2}$ is a LEC special to Wilson fermions and arises because of chiral symmetry breaking. Thus, it appears only in the sea sector. The LEC $c_{1}$ is special to the mixed-action case, as can be seen from the fact that it disappears from $V$ when we replace $P_{s}$ by the unit matrix. Both $c_{1}$ and $c_{2}$ are lattice artifacts, containing a factor $a^{2}$. There are several more terms in the chiral potential $V$, but those do not affect the discussion of the phase diagram we are interested in here, and we therefore omit them in this talk.

Since we will always keep $m_{v}>0$ in our discussion below, all the chiral symmetries are explicitly broken (softly in the valence sector), and the full symmetry group $G$ is a generalization of isospin,

$$
G=U(2)_{\text {sea }} \times U(2 \mid 2)_{\text {valence }} .
$$

At non-zero lattice spacing there are no symmetries relating the sea and the valence-ghost sectors, because of the different choices for the sea and valence Dirac operators [3]. Within each sector we will always maintain isospin symmetry, taking the up and down quarks degenerate in each sector.

\section{A puzzle}

Let us expand $V$ around the trivial vacuum in order to obtain the pion masses at leading order in ChPT (taking $m_{s, v}>0$ ):

$$
\begin{aligned}
& M_{s s}^{2}=2 B_{0}\left(m_{s}+4 c_{2}\right), \\
& M_{v v}^{2}=2 B_{0} m_{v}, \\
& M_{s v}^{2}=B_{0}\left(m_{s}+m_{v}+4 c_{1}+2 c_{2}\right),
\end{aligned}
$$

with $M_{s s}$ the mass of a pion made out of two sea quarks, $M_{v v}$ the mass of a pion made out of two valence quarks, and the mixed-pion mass $M_{s v}$ of a pion made out of a sea and a valence quark.

The third line of Eq. (3.1) suggests that spontaneous symmetry breaking (SSB) can take place if $2 c_{1}+c_{2}<0$, because in that case decreasing the sum $m_{s}+m_{v}$ makes $M_{s v}^{2}$ negative. This would drive the theory into a "mixed phase," with a condensate $\left\langle\bar{q}_{s} \gamma_{5} q_{v}\right\rangle \neq 0$. Indeed, if we choose $c_{2}>0$ (to avoid the complications of an Aoki phase [9] in the sea sector [8]), but $2 c_{1}+c_{2}<0$, minimization of the potential $V$ leads to the conclusion that for $m_{s}+m_{v}+4 c_{1}+2 c_{2}<0$ the theory enters a mixed phase.

The mixed condensate would break the symmetry group $G$ down to a diagonal group in which the remaining sea and valence quark symmetry transformations are the same. Sea and valence sectors mix, and this has consequences for the spectrum of the theory. An interesting example is

\footnotetext{
${ }^{1}$ Terms linear in $a$ can be absorbed into the quark masses [8].
} 
the two-point function $\left\langle\pi_{s s}^{+}(0) \pi_{s S}^{-}(t)\right\rangle$, which is made out of sea pions only, but which in the mixed phase becomes dependent not only on $m_{s}$, but also on $m_{v}{ }^{2}$

This, however, creates a paradox: by the very construction of MAQCD, this can never happen! In an actual simulation, gauge-field configuration depend only on $m_{s}$, or, in other words, the dynamics of the theory can only depend on $m_{s}$. In particular, if one considers a correlation function that depends only on sea fields, such as $\left\langle\pi_{s s}^{+}(0) \pi_{s s}^{-}(t)\right\rangle$, one performs a simulation in the theory with only sea quarks; no valence quarks are present anywhere in such a computation! $!^{3}$

If this were a true paradox, this would imply that the field-theoretical description of MAQCD is fatally flawed, and, as a consequence, that standard EFT techniques cannot be used to interpret results obtained in a mixed-action simulation. This state of affairs leads to the following important questions:

1. Does MAQCD, in its field-theoretical definition, indeed have a mixed phase, i.e., a phase with $\left\langle\bar{q}_{s} \gamma_{5} q_{v}\right\rangle \neq 0$ (as appears to be predicted by MAChPT)?

2. If not, does MAChPT get it wrong?

In the following section we will see that in fact this dangerous scenario cannot occur, and that MAChPT is forced, by the underlying theory, to get it right.

\section{Resolving the puzzle}

We begin with a theorem that holds in the underlying theory, MAQCD with two sea quarks and two valence quarks, invariant under the isospin group $G$ of Eq. (2.4). ${ }^{4}$ The theorem states that no spontaneous isospin breaking can occur in the valence sector. This is an almost direct consequence of the well-known Vafa-Witten [10] theorem, that forbids breaking of vectorlike symmetries in the continuum. The theorem extends to the valence sector because even in the lattice theory the valence quarks are chiral; the Vafa-Witten theorem applies to any type of Ginsparg-Wilson quarks. ${ }^{5}$

An immediate consequence is that a mixed phase cannot occur, because such a phase would break $U(2)_{\text {sea }} \times U(2)_{\text {valence }} \rightarrow U(2)_{\text {diagonal }}$, and thus a non-zero value of $\left\langle\bar{q}_{s} \gamma_{5} q_{v}\right\rangle$ would break $U(2)_{\text {valence }}$, in contradiction with the Vafa-Witten theorem.

This answers the first of the two questions raised in the previous section: MAQCD cannot have a mixed phase, and the paradox cannot occur. But, this still leaves open the second question: what about MAChPT?

\section{A mass inequality}

By choosing $m_{s}>0$ and $m_{v}>0$ large, we can arrange that $M_{s s}^{2}, M_{v v}^{2}$, and $M_{s v}^{2}$ are all strictly

\footnotetext{
${ }^{2}$ As can be demonstrated explicitly by a somewhat tedious but straightforward calculation.

${ }^{3}$ The paradox persists in finite volume, because $\left\langle\pi_{s s}^{+}(0) \pi_{s s}^{-}(t)\right\rangle$ is invariant under integration over the orientation of $\left\langle\bar{q}_{s} \gamma_{5} q_{v}\right\rangle$ in the mixed phase.

${ }^{4}$ In fact, this theorem holds for any number of valence quarks.

${ }^{5}$ It can also be shown that the theorem extends to the ghost sector, and, in particular, that no Grassmann-valued condensates can occur [6].
} 
positive, so that no isospin breaking takes place in the EFT. Then, from the identity

$$
\operatorname{tr}\left\langle\left(S_{s d}(x, y)-S_{v d}(x, y)\right)^{\dagger}\left(S_{s d}(x, y)-S_{v d}(x, y)\right)\right\rangle \geq 0
$$

in the underlying theory, where $S_{s i}\left(S_{v i}\right)$ is the sea (valence) quark propagator for flavor $i=u, d$, and $S_{d}^{\dagger}(x, y)=\gamma_{5} S_{u}(x, y) \gamma_{5}$ for both sea and valence propagators, it follows that

$$
\left\langle\pi_{s s}^{+}(x) \pi_{s s}^{-}(y)\right\rangle+\left\langle\pi_{v v}^{+}(x) \pi_{v v}^{-}(y)\right\rangle \geq\left\langle\pi_{s v}^{+}(x) \pi_{v s}^{-}(y)\right\rangle+\left\langle\pi_{v s}^{+}(x) \pi_{s v}^{-}(y)\right\rangle
$$

Translating this to ChPT, this inequality implies that

$$
M_{s v} \geq \min \left(M_{s s}, M_{v v}\right)
$$

Let us now argue that this inequality restores the validity of MAChPT. From Eq. (3.1), we have that

$$
M_{s v}^{2}=\frac{1}{2}\left(M_{s s}^{2}+M_{v v}^{2}\right)+2\left(2 c_{1}-c_{2}\right) .
$$

Choosing quark masses such that $M_{s s}=M_{v v}$, the inequality (5.3) implies that $M_{s v} \geq M_{s s}=M_{v v}$, and thus, using Eq. (5.4), that

$$
2 c_{1}+c_{2} \geq 2 c_{1}-c_{2} \geq 0
$$

(recall that we chose $c_{2}>0$ ). But, now we can turn this around, using the fact that the LECs $c_{1}$ and $c_{2}$ are independent of the quark masses $m_{s}$ and $m_{v}$, so that inequality (5.5), together with the third equation in Eq. (3.1), implies that always, irrespective of the value of the quark masses, $M_{s v}^{2} \geq 0$, and no SSB to a mixed phase can occur, also in MAChPT. In words, the mass inequality (5.3) restricts the values of the LECs in the EFT such that the EFT is forced to faithfully reproduce the phase structure of the underlying theory. While we demonstrated this here for the simplified potential of Eq. (2.2), and for the case $c_{2}>0$, it is clear that, in general, the parameters of MAChPT are restricted such that regions in the phase diagram with a mixed condensate are excluded, because of the fact that a mixed phase cannot occur in the underlying theory.

To summarize the situation, the Vafa-Witten theorem restricts the vacuum expectation value of the non-linear field to the form

$$
\Sigma_{\text {vacuиm }}=\left(\begin{array}{ccc}
\Sigma_{s s} & \mathbf{0} & \mathbf{0} \\
\mathbf{0} & \mathbf{1} & \mathbf{0} \\
\mathbf{0} & \mathbf{0} & \mathbf{1}
\end{array}\right)
$$

where $\Sigma_{s s}$ is the $2 \times 2$ unitary matrix describing the vacuum in the sea sector, $\mathbf{0}$ is the $2 \times 2$ null matrix, and 1 is the $2 \times 2$ unit matrix. Substituting this form into $V$, the potential reduces to

$$
V=-m_{s} \operatorname{tr}\left(\Sigma_{s s}+\Sigma_{s s}^{\dagger}\right)-\frac{1}{2} c_{2}\left(\operatorname{tr}\left(\Sigma_{s s}+\Sigma_{s s}^{\dagger}\right)\right)^{2} .
$$

This is precisely the Sharpe-Singleton potential for the sea sector, which predicts the Aoki phase for $c_{2}<0$. No other non-trivial phase can occur. 


\section{Conclusion}

Referring back to the two questions raised toward the end of Sec. 3, the answer to the first question is no, MAQCD cannot have a mixed phase. Because the valence quarks are not part of the dynamics, the phase structure is restricted to that of the sea sector alone. In our case, this implies that the only possible non-trivial phase is an Aoki phase. The answer to the second question is also no. In other words, MAChPT gets it right. As we have seen, mass inequalities in the underlying theory restrict the values of LECs in the EFT such as to make regions in the phase diagram with a mixed phase inaccessible. We gave an example of such a restriction, and it is possible that more such constraints on the LECs exist. These would be uncovered by a study of the full chiral effective potential for arbitrary $\Sigma_{\text {vacuum }}$ by imposing restrictions following from the Vafa-Witten theorem in the underlying theory.

One might ask whether a similar argument could lead to a restriction on the value of $c_{2}$, which, for negative values leads to the existence of the Aoki phase [8]. The answer is negative, because the argument would have to involve the neutral pion, and thus (quark-)disconnected diagrams not captured by the expression in Eq. (5.1). Finally, we note that our conclusions presumably generalize to other mixed-action theories, such as those with a staggered sea sector and a chiral valence sector [11].

\section{Acknowledgements}

MG thanks the Department of Physics of Humboldt Universität zu Berlin, and YS thanks the Department of Physics and Astronomy of San Francisco State University for hospitality. OB is supported in part by the Deutsche Forschungsgemeinschaft (SFB/TR 09), MG is supported in part by the US Department of Energy, and YS is supported by the Israel Science Foundation under grant no. $423 / 09$.

\section{References}

[1] C. Bernard and M. Golterman Phys. Rev. D 49, 486 (1994) [arXiv:hep-lat/9306005].

[2] O. Bär, G. Rupak and N. Shoresh, Phys. Rev. D 70, 034508 (2004) [arXiv:hep-lat/0306021].

[3] O. Bär, G. Rupak and N. Shoresh, Phys. Rev. D 67, 114505 (2003) [arXiv:hep-lat/0210050].

[4] M. Golterman, T. Izubuchi and Y. Shamir, Phys. Rev. D 71, 114508 (2005) [arXiv:hep-lat/0504013].

[5] P. H. Ginsparg and K. G. Wilson, Phys. Rev. D 25, 2649 (1982).

[6] O. Bär, M. Golterman and Y. Shamir, Phys. Rev. D83, 054501 (2011) [arXiv:1012.0987 [hep-lat]].

[7] M. Golterman, in “Modern Perspectives in Lattice QCD," eds. L. Lellouch et al. (Les Houches 2009), Oxford [arXiv:0912.4042 [hep-lat]].

[8] S. R. Sharpe and R. L. Singleton Jr., Phys. Rev. D 58, 074501 (1998) [arXiv:hep-lat/9804028].

[9] S. Aoki, Phys. Rev. D 30, 2653 (1984).

[10] C. Vafa and E. Witten, Nucl. Phys. B 234, 173 (1984).

[11] O. Bär, C. Bernard, G. Rupak and N. Shoresh, Phys. Rev. D 72, 054502 (2005) [arXiv:hep-lat/0503009]. 